\title{
Formation au travail de l'erreur et didactique des mathématiques
}

\section{Annick Flückiger}

Le développement des théories constructivistes a modifié le rapport à l'erreur tel qu'il apparaît en lien avec le développement des connaissances. Les théories didactiques en général et la didactique des mathématiques en particulier ont participé à ce mouvement. Cet article donne à voir comment cette dernière a contribué à cette évolution et comment certains des concepts qu'elle a développé au cours des trente dernières années, permettent d'enrichir la problématique de l'erreur relativement à l'enseignement apprentissage des mathématiques à l'école. Sur la base de données recueillies auprès d'étudiants en formation d'enseignants, est mis en évidence le décalage qui existe entre le discours usuel qui prend en compte la perspective constructiviste d'une part et les pratiques réelles du traitement de l'erreur d'autre part. L'article pose alors le problème d'une formation des enseignants qui permettrait de faire évoluer ces pratiques et propose quelques orientations pour penser cette formation.

\section{Introduction}

En même temps que se sont développées les théories didactiques, s'est spécifié un certain type de rapport à la fois à l'erreur et au travail de l'erreur. Il s'agit, par l'utilisation de cette expression ${ }^{1}$, de rappeler que dans le contexte scolaire, l'erreur n'est évidemment pas associée au seul élève mais que, dans une perspective didactique, l'erreur -sa production ou son absence- est à rapporter aux trois soussystèmes du système didactique, système modélisé par le triplet (maître, élève, savoir). Quelles sont les postures et/ou actions du maître et de l'élève face à l'erreur ? Quelles interactions sont engendrées ou modifiées ? Quel lien est à faire avec le savoir en jeu ? Quel contrat didactique permet ou non un travail de l'erreur adéquat pour les apprentissages ?... Les questions sont multiples et nous traiterons plus spécifiquement ici la question de la formation des enseignants. Ce que nous tenterons de mettre en évidence dans cet article est la nécessité de changer, par le biais de la formation, le positionnement épistémologique et in fine les pratiques des enseignants relativement à la question de l'erreur dans l'enseignement / 
apprentissage des mathématiques. En effet un pas important reste à faire pour que se traduisent dans les pratiques réelles les prises de position initiées par les recherches en didactique, positions souvent intégrées au niveau du discours par les professionnels de l'enseignement et diffusées au niveau des injonctions que reçoivent les enseignants.

Notons d'entrée que notre positionnement théorique se réfere à la didactique des mathématiques francophones ${ }^{2}$ telle qu'elle s'est développée au cours des trente dernières années avec la théorie des situations de Brousseau (1986; 1998), la théorie des champs conceptuels de Vergnaud (1991) et, plus récemment, la théorie anthropologique du didactique de Chevallard (1999). Sans prétendre à l'exhaustivité, nous tenterons dans cet article de donner à voir notre univers de référence et de pointer quelques concepts pertinents permettant d'alimenter la réflexion sur la problématique de l'erreur dans l'enseignement apprentissage des mathématiques en soulignant quelques apports spécifiques de la didactique des mathématiques quand il s'agit de la question de l'erreur.

Suivant en cela les préoccupations de Brousseau, nous nous focaliserons ensuite sur l'important problème de la formation des enseignants.

La communauté a peine à reconnaitre que l'activité propre de celui qui apprend (élève ou institution), a parfois pour résultat des erreurs légitimes, et même la constitution d'obstacles, quelle que soit la qualité de l'enseignement reçu. Les mathématiciens, et le public ont du mal à accepter ces écarts autour du "vrai» savoir, et les professeurs ont du mal à les prendre en charge autrement que comme des fautes. Tous peinent enfin à croire que ces phénomènes ne seront pas régulés efficacement tant que l'on ne possédera pas les connaissances spécifiques nécessaires, [.....] et que vraisemblablement elles devront figurer dans la formation des professeurs de mathématiques (Brousseau, 2003).

Dans la deuxième partie, nous présenterons, sur la base d'une enquête et d'observations menées récemment auprès d'enseignants en formation, le positionnement «classique» et spontané relativement à la question de l'erreur. Ces résultats permettent de mettre en évidence l'ampleur des besoins auxquels la formation doit essayer de répondre.

La troisième partie donnera à voir les résultats d'une recherche menée en didactique des mathématiques quant à la formation au travail de l'erreur de futurs enseignants. En lien avec d'autres concepts développés dans le même univers théorique, il sera alors possible de proposer des pistes nouvelles de réflexion quant à la question qui nous occupe ici.

\section{Erreur et didactique des mathématiques}

Lorsqu'il s'agit du monde scolaire, de l'enseignement, l'erreur est au cœur des interactions entre enseignant et élève, c'est ce qui est souligné dans le texte ci-dessous: 
L'enfant lorsqu'il entre en classe, devient un élève. C'est-à-dire un sujet soumis à des règles et des lois qui sont, en ce lieu, essentiellement didactiques. Il entre dans un monde où règne un ordre, un ordre didactique: on y vient en particulier pour essayer de ne pas "se tromper».

Combien seraient bouleversés les rapports dans la classe, si l'élève, à l'instar du maître idéal, ne fournissait plus d'erreurs! Quelle serait la substance, la matière première, à partir de laquelle se construirait, avec le maitre le dialogue sur l'apprentissage? L'erreur, par le discours qu'elle provoque, par le message qu'elle renvoie, par les repères qu'elle crée, est bel et bien le principal vecteur de la communication sur ce qui façonne pour l'essentiel la relation didactique: enseigner, apprendre.. .et montrer ce que l'on sait (Ravenstein \& Sensevy, 1993-1994, p. 83).

Dès son origine la didactique des mathématiques s'est emparée de ce sujet, didactifiant la question du rapport à l'erreur, la question de l'analyse et du traitement des erreurs des élèves -voire celles des maîtres- dans les situations de classe. Le tout premier numéro de la revue Recherches en Didactique des Mathématiques se présente en 1980 comme le lieu d'expression et de débat pour une nouvelle communauté de chercheurs s'attachant à «cerner les faits spécifiques de l'enseignement et en tant qu'il s'agit d'une discipline déterminée, donc en tant qu'il se réalise effectivement dans une classe, avec des élèves, un maître, une connaissance». (Rouchier, 1980, p. 7). Rouchier présente dans ce premier numéro le projet de ce courant qui vise à «élaborer une théorie des situations aménagées dans un but d'enseignement des mathématiques et une théorie des processus d'acquisition». Il cite alors, parmi les objectifs des recherches expérimentales concernées, celles portant sur l'analyse des conduites ${ }^{3}$ et des productions des élèves au cours d'un apprentissage. Dès le début donc, l'analyse des erreurs est une composante affirmée des recherches en didactique.

Dans ce même numéro, Brousseau traitant du «Problème de l'enseignement des décimaux» oppose deux approches des problèmes relatifs à cet enseignement. Il les différencie en faisant référence à la question de l'erreur. Dans la première dit-il:

L'erreur est interprétée comme l'indice du non-fonctionnement d'une des séquences prévues, d'un écart entre un projet et sa réalisation: le savoir a été mal appris, mal retenu, ou mal appliqué ou mal compris, mal relié aux autres savoirs...Les difficultés sont celles de l'élève ou celles de l'enseignant,..... ou celles de l'enseignement: les objectifs sont mal communiqués, les moyens sont insuffisants, les résultats mal évalués etc. (Brousseau, 1980, p. 51).

En opposition à cette approche, Brousseau en identifie une autre qui, se référant à Piaget et à Bachelard, présente un point de vue différent sur l'erreur:

L'apprentissage est une adaptation de l'élève à une situation problème nouvelle. Les difficultés qu'il rencontre sont donc fondamentales pour provoquer cette adaptation. De plus elles peuvent être constitutives du nouveau savoir c'est-à-dire être indispensables à sa compréhension. Ces difficultés sont celles qui portent en elles la 
conception antérieure de l'élève et la situation-problème choisie les a seulement révélées (Brousseau, 1980, p. 51).

Cette position d'origine constructiviste, au fondement de la didactique des mathématiques, place l'erreur au cœur du processus d'adaptation et donc de l'apprentissage dans les théories modernes qui veulent que les connaissances se "construisent». Modélisant le fonctionnement du système didactique en termes de situations (d'action, de formulation, de validation et d'institutionnalisation), Brousseau établit dans ce cadre théorique la nécessité d'une connaissance des erreurs, celles effectivement observées et celles possibles. Il précise deux critères déterminants pour une analyse didactique: «Une erreur doit être rapportée à un moment précis d'une situation et à une connaissance ou à un savoir déterminés qui permettent de la concevoir» (Brousseau, 2000/2001, p. 8).

Il différencie dans ce cadre théorique, les erreurs dites de décision et relatives à l'action en cours, les erreurs de formulation dans les jeux de communication, les erreurs d'interprétation, de compréhension, d'explication qui apparaissent au cours des débats, erreurs qui se réferent à la conception des élèves et au savoir.

Dès le début sont mis en place les ingrédients qui vont constituer les fondamentaux du rapport à l'erreur dans une perspective didactique: situation, savoir, débat, conception en sont les éléments clefs.

La situation est au cœur des modélisations didactiques initiées par Brousseau. En accord avec ce positionnement théorique, Margolinas souligne le lien entre statut de l'erreur et situation dans laquelle est engagé l'élève: «Dans la classe le statut de l'erreur change selon que l'élève est dans une situation d'apprentissage par adaptation ou dans une situation de réinvestissement. Ce statut est lié également à la conception de l'apprentissage, constructiviste ou empiriste» (Margolinas, 1993, p. 170).

Ce statut de l'erreur, l'auteur le renvoie également à la conception de l'apprentissage, nous reviendrons sur cet aspect.

Dans un texte intitulé «Statuts de l'erreur dans la relation didactique» et dont nous avons déjà cité l'introduction, Ravenstein et Sensevy (1993-1994), se référant à des travaux issus de disciplines voisines différencient:

- l'erreur comme mesure d'écart, rapport à la norme,

- de l'erreur décisionnelle, soulignant "qu'il n'y a d'erreur que s'il y a possibilité de choix et s'il existe des critères de distinction de ces choix» (Leplat \& Pailhous, 1974), cité par Ravenstein et Sensevy.

Cette distinction est intéressante car elle discrimine, ainsi que le souligne les auteurs:

- ce qui, dans la relation didactique concerne la partie publique du rapport au savoir de l'élève mais qui ne peut fournir les informations nécessaires à l'activité réelle de l'élève au travail,

- de ce qui concerne «le rapport privé de l'élève au savoir, c'est-à-dire sa manière très personnelle, unique, de s'approprier ce qu'on lui enseigne» (a.a.O.). 
L'action du maître le conduit à attribuer un sens à l'erreur repérée. Selon que l'erreur est isolée, massive, ponctuelle, fréquente etc., les causes attribuées à sa production et les actions engagées par l'enseignant pour son traitement vont être différentes. Ravenstein et Sensevy soulignent que les erreurs prises en compte par les maîtres appartiennent exclusivement à la première catégorie, celles relatives à la partie publique du rapport au savoir de l'élève, celles qui seront traitées par modification de la tâche proposée ou les conditions de sa réalisation. Or c'est bien dans la deuxième dimension citée qu'il y a problème, tant du point de vue du repérage de l'erreur que de son traitement. Il est clair que prendre en compte les erreurs du deuxième genre pose un problème d'une autre envergure, celui de la prise en compte des stratégies des élèves. Nous utilisons le terme de stratégie $e^{4}$ dans une acception qui lie stratégie à situation. Pour nous ce n'est pas relativement à la tâche qu'il y a stratégie mais relativement à la situation, situation qui active ou inhibe certains schèmes. Prendre en compte les stratégies des élèves, suppose en amont de la séquence d'enseignement un travail d'analyse des contenus et d'identification des erreurs potentielles ainsi qu'un positionnement théorique permettant l'interprétation des erreurs observées. Ceci ne fait pas partie de la panoplie professionnelle usuelle, c'est cette panoplie qu'il faut enrichir au cours de la formation.

La perspective didactique impose une centration à la fois sur la situation et sur le savoir en jeu et donc sur le contrat qui cimente à un moment donné, les relations entre les trois pôles du système didactique. Différents concepts développés, participant à la théorisation didactique, permettent de renouveler et d'enrichir le questionnement relatif à l'erreur. Prenons l'exemple du concept de dévolution.

S'il est admis comme une évidence que faire des mathématiques c'est résoudre des problèmes et qu'apprendre les mathématiques consiste à être confronté à des problèmes adéquats, le débat sur ce qu'est un problème reste vif. En classe et conformément aux théories en vigueur, pour apprendre, l'élève doit être confronté à des problèmes dont la résolution va le conduire à développer des stratégies pertinentes pour l'apprentissage. L'une des questions qui se pose est celui de la dévolution. La dévolution est, en théorie didactique, une condition d'accès au sens de la connaissance. Formellement elle est définie par Brousseau comme: «'acte par lequel l'enseignant fait accepter à l'élève la responsabilité d'une situation a-didactique ${ }^{5}$ ou d'un problème et accepte lui-même les conséquences de ce transfert» (Brousseau, 1990, p. 325).

A la lueur de ce concept, Ravenstein et Sensevy déjà cités et à la suite d'Amigues (1991), posent alors ainsi la question de l'erreur:

L'erreur peut provenir directement du fait que le problème posé n'est pas un problème pour l'elève: il ne fournit donc pas de solution, ou fournit une solution erronée én conformité par rapport au contrat didactique. Il nous faut donc admettre avec Brousseau l'importance de la dévolution du problème, admettre que l'élève doit se faire sujet de la question qui lui est posée (Ravenstein \& Sensevy 1993-1994, p. 86). 
Ils rapportent cela à l'idée plus générale que, en situation scolaire, les tâches ne sont pas vraiment là pour être faites mais qu'elles ont une fonction ostensive, «on fait pour montrer que l'on a appris» disent-ils. Notons que le concept de dévolution, très largent diffusé dans la communauté enseignante, est souvent interprété comme l'acte qui consiste pour le maître à lire la consigne et à ne plus intervenir. Brousseau (1990) affirme que de nombreux problèmes analysés en termes «d'absence de motivation des élèves», gagneraient à être pensés relativement à ce concept de dévolution.

Travaillant cette question, Ratsimba-Rajohn (1992), met en évidence que l'élève doit prendre conscience d'une opposition entre la situation a-didactique mise en place par l'enseignant et le modèle de situation intégré dans ses propres savoirs. C'est à cette condition dit-il que peut s'amorcer le processus de dévolution. Les oppositions et les contradictions qui cohabitent chez un individu ou à l'intérieur de la classe vont produire des affirmations, des prises de position et des décisions contradictoires lors des débats. Leur repérage, leur identification et leur traitement est un aspect du travail de l'enseignant qui nous semble essentiel quand il s'agit de penser le rapport à l'erreur dans l'enseignement. Nous reviendrons sur cet aspect essentiel.

Ces éclairages issus de la recherche en didactique restent encore bien souvent pour le moment le fait des chercheurs, sans réelle prise en compte dans les pratiques enseignantes. La question qui nous voulons poser ici est celle de la formation des futurs enseignants. Que faut-il introduire dans la formation des enseignants, à la fois comme concept théorique et comme pratiques liées à l'erreur pour faire évoluer les comportements professionnels relatifs à la question de l'erreur? Portugais (1995) a mis en pratique et étudié un dispositif de formation prenant en compte cette dimension, nous présenterons les aspects liés au travail de l'erreur de cette recherche dans la troisième partie.

Auparavant, à l'aune des apports théoriques mentionnés ci-dessus, pointons les besoins qui se font jour relativement à la formation des enseignants et ce, sur la base d'une enquête menée récemment.

\section{Rapport à l'erreur chez les étudiants en formation}

\section{Un discours}

L'enquête a été conduite auprès d'un groupe d'étudiants de la section des sciences de l'éducation à l'université de Genève, étudiants inscrits dans le cursus dit LME (Licence Mention Enseignement) cursus destiné à former dans une alternance cours théoriques/temps de terrain, les futurs enseignants de l'enseignement primaire genevois. Au moment de cette étude, ces étudiants étaient dans leur troisième année universitaire et dans leur deuxième année de formation à 
l'enseignement. La question ci-dessous leur a été posée au retour d'un stage dans une classe: Qu'est ce que c'est pour vous une erreur dans le cadre de l'enseignementapprentissage des mathématiques?

Sans traiter en détail l'ensemble des données recueillies, plusieurs remarques sont à faire. L'une d'elle concerne le lien fait entre erreur et savoir. En effet, bien que d'une part la question ait été posée au cours d'un séminaire de didactique des mathématiques et que, d'autre part, elle ait été centrée explicitement sur l'enseignement - apprentissage des mathématiques, le renvoi au contenu de savoir est quasi absent des réponses des étudiants. Seuls deux étudiants sur vingt-cinq y font référence, l'un pour hiérarchiser les erreurs en fonction du type d'activité en jeu:

Une erreur est selon moi plus grave lorsque celle-ci se perçoit dans une démarche incorrecte, dans un mauvais raisonnement que dans une petite faute de calcul, bien que cette dernière soit également une erreur,

l'autre pour exemplifier une étape de l'apprentissage des soustractions en faisant référence à une erreur à la fois fréquente et significative:

dans la soustraction de 11-9 en colonne, faire 9-1 =8 et 1 - rien égal 1 donc 18 (sic) Excepté dans ces deux cas, le discours produit reste très général, en dehors de toute prise en compte du contenu enseigné. Or, nous avons vu que dans les travaux des didacticiens la dimension relative à la prise en compte de la connaissance (ou du savoir) est un des éléments clés à mettre en relation avec les erreurs en situation de classe.

Il est à noter que cette absence de prise en compte du savoir n'est pas le seul fait des étudiants en formation initiale. En effet Comiti et Grenier (1991) font la même observation en interrogeant des professeurs de mathématique exerçant dans le secondaire (articulation collège-lycée en France).

Dans les réponses relevées au cours de l'enquête auprès des étudiants en formation, la perspective constructiviste, actuellement valorisée dans le discours tenu sur l'enseignement, oriente les prises de position relativement à la question de l'erreur. Citons quelques propos représentatifs:

- l'erreur est "normal», "naturel» nécessaire utile quel que soit le type d'erreur (sic)

- elle permet l'apprentissage

- l'erreur a un rôle fondamental puisqu'elle permet à l'élève de progresser dans ses apprentissages

En quoi et comment cette approche de l'erreur est-elle opérationnalisée? Comment ces prises de position "théoriques» se traduisent-elles dans l'action? Les réponses des étudiants de l'enquête donnent à voir quelques axes de réflexion sur ce que signifie pour eux, une prise en compte des erreurs dans le quotidien de la classe. Tout d'abord avant de traiter l'erreur il s'agit de l'analyser, de lui attribuer un statut, toutes les erreurs ne seront pas gérées de façon identique:

L'erreur peut se gérer différemment selon son niveau de gravité et sa répétition. De plus elle ne sera pas gérée de la même façon si elle se retrouve chez un seul élève ou chez toute la classe 
Nous retrouvons certaines des catégories mises à jour dans les travaux en sciences de l'éducation (voir notamment Astolfi, 1997). Les étudiants l'affirment, pour l'enseignant, l'erreur est une source d'information qui lui permet de réguler son action:

Une erreur c'est un point de départ pour faire avancer l'élève, un point de repère pour l'enseignant, une remise en question de l'enseignant et de l'enseignement

En particulier, l'erreur permet d'établir un état d'avancement de l'apprentissage de l'élève

Les erreurs permettent de voir où en sont les élèves dans leur apprentissage.

Et au-delà de cette information sur la chronogenèse ${ }^{6}$ des savoirs, un étudiant voit dans l'erreur la possibilité d'avoir une donnée plus «interne» de la genèse des connaissances en accédant au fonctionnement cognitif:

Les erreurs permettent parfois de comprendre les stratégies de l'élève et son fonctionnement cognitif

La grande prudence de cette affirmation est tout à fait pertinente. Comprendre les stratégies, parfois, est bien un des buts qui peut être assigné à un travail de repérage et d'interprétation des erreurs, mais il demande une capacité d'analyse qui doit justement être l'objet de la formation et n'a rien de spontané.

Le débat se donne souvent à voir comme une réponse adéquate à la production d'erreur dans la gestion de la classe:

[l'erreur] peut être débattue en classe entière et permettre ainsi à d'autres élèves de partager ce qu'ils pensent de leurs stratégies.

Certains nuancent, ne valorisent le travail collectif de l'erreur que pour autant que cela ait un sens dans la prise en compte du temps didactique, dans l'avancement collectif du temps du savoir:

C'est important de rebondir sur l'erreur mais qu'elle puisse faire avancer le «smilblick»

Notons encore à propos de cette enquête que les erreurs sont à la fois annoncées comme "normales», mais qu'elles sont en fait traitées davantage comme des $d y s$ fonctionnements que comme des étapes, dysfonctionnement chez l'élève qui n'a pas compris, dysfonctionnement de l'enseignement:

Une erreur c'est un dysfonctionnement de la mémoire

Les erreurs montrent à l'enseignant les points qu'il doit reprendre dans son enseignement

C'est d'ailleurs le terme de «remise en question» qui est le plus utilisé, il est même omniprésent dans les réponses analysées:

Une erreur c'est une remise en question de l'enseignant et de l'enseignement

Pour autant qu'elle soit bien gérée, l'erreur conduit à cette remise en question:

Selon la place accordée à l'erreur l'enseignant peut amener ses élèves à se poser des questions, à se remettre en question.

Une erreur c'est formatif, elle permet de se remettre en question, de réfléchir afin de trouver la bonne réponse. 
Cette confiance exprimée est en cohérence avec une pratique pédagogique courante qui consiste à faire travailler les élèves sur leurs productions erronées. Or un récent travail de mémoire de licence (Buchli, 2005) centré sur les erreurs des élèves et la lecture qu'eux-mêmes peuvent en faire a mis en évidence un résultat tout à fait intéressant. Sur un sujet didactiquement dépassé, seuls les élèves ayant un statut scolaire que l'auteure qualifie de "fort", sont à même d'analyser les productions erronées qui leur sont proposées en faisant référence au contenu de savoir. Les autres élèves, à défaut d'identifier le pourquoi de l'erreur en termes de contenu, se centrent sur les caractéristiques de l'apprenant. L'auteure pose alors, à juste titre, le problème de la pertinence de cette pratique scolaire qui consiste à donner à corriger aux élèves leurs propres erreurs. Compte tenu de ce résultat de recherche, il semble utile de se poser la question des conditions qui rendent cette pratique pertinente pour les apprentissages, pertinence qui suppose que l'adaptation de l'élève au contrat ne soit pas la seule cause des décisions prises lors de l'activité de correction.

\section{Des pratiques}

Il est intéressant de mettre brièvement en relation les réponses obtenues lors de cette enquête avec d'autres données relatives à des étudiants du même cursus mais dans un autre contexte. Il s'agit dans ce cas, pour les étudiants, de conclure un travail ayant trait à une analyse des erreurs relevées suite à une passation d'épreuve de mathématique dans une classe. Dans cette conclusion, les étudiants qui terminent leur stage, doivent garder leur positionnement d'enseignant et anticiper une séance "post» en lien avec les erreurs observées.

Deux remarques sont à faire. La première peut se traduire en termes de désarroi face aux productions erronées des élèves, notamment pour les interpréter:

Comment savoir à partir des données écrites seulement et non de la pensée de l'élève, si l'interprétation que l'on fait de ses erreurs est conforme à ce qui sest réellement passé. Parfois l'interprétation est unique, par contre je me suis souvent trouvée dans la situation où je pouvais voir plusieurs pistes différentes de procédures conduisant à l'échec.

Comment se fait-il que certains élèves n'arrivent pas à comprendre des problèmes simples? est-ce parce qu'ils n'arrivent pas à comprendre le problème ou vraiment ne sont-ils pas capables de les comprendre? et dans ce cas comment peut-on aider un élève à comprendre les problèmes ? j’imagine qu'il faudrait prendre un tel enfant seul, lui demander de bien lire le problème et de le réexpliquer.

La deuxième observation est que, dans ce type d'écrit, se donnent à voir les théories implicites relatives à la construction des connaissances et leurs conséquences sur les pratiques supposées pertinentes pour l'enseignement:

Il me semblerait utile de reprendre le problème ensemble, avec toute la classe, et de mettre à plat les étapes importantes. Pour cela, un exercice relatif à la lecture des 
consignes serait utile [..] un autre point important à travailler est la division et la multiplication des nombres décimaux, insister sur la place de la virgule et sur l'importance du zéro. De plus jélargirais le problème aux puissances de dix [...] Ensuite je pense qu'un travail sur l'organisation d'un tableau serait indispensable. Pour terminer, une fois que toutes les difficultés auront été abordées, je ferais refaire l'exercice (en changeant les données) pour me rendre compte de ce qui est acquis et ce qui est encore à travailler

Il serait judicieux d'envisager de leur faire travailler les différentes opérations avec des nombres décimaux, afin que petit à petit, les élèves apprennent à les manipuler dans les algorithmes d'addition, puis de soustraction. Ceci permettrait dans un temps plus tardif de reprendre les multiplications des décimaux afin de noter sily a eu progression ou non.

Il est clair que si les thèses constructivistes façonnent singulièrement les prises de position a priori sur la prise en compte des erreurs, d'autres postures se font jour lorsqu'il s'agit de quitter cette position de principe et de prendre une décision professionnelle en lien avec une classe réelle. Ce qui se donne alors à voir est une épistémologie professionnelle pour laquelle le savoir est à la fois linéaire et cumulatif. Dans cette optique, il s'agit de restaurer pour les élèves un rapport au savoir adéquat par des retours en arrière permettant des reprises, des découpages plus fins des difficultés, bref une épistémologie plus proche du béhaviorisme que du constructivisme.

Notons que Brun et Conne (1993) font les mêmes observations relativement à des enseignants en formation continue. Ces enseignants expérimentés face à une production erronée d'élève proposent systématiquement une reprise de l'explication et parmi les propositions d'action, ils proposent de "faire s'interroger les élèves sur leur calcul», ce que les auteurs traduisent en terme de "remise de la question dans le camp de l'élève». D'une façon générale, disent les chercheurs cités, «il peut sembler raisonnable de ne pas s'en remettre à la bonne explication qui lèverait enfin les obstacles à l'apprentissage».

La question qui se pose est bien de dépasser les positions de principe en vigueur dans la profession et de trouver des éléments de réponse aux deux questions suivantes:

- comment faire en sorte que la prise en compte des erreurs soit effective et pertinente dans la gestion professorale de l'enseignement - apprentissage des mathématiques,

- et en amont, quelle formation pour les futurs enseignants est susceptible de conduire à cela ?

C'est à la question de la formation initiale que nous tentons ci-dessous, d'apporter des éléments de réponse. 


\section{Travail de l'erreur et formation initiale des enseignants}

La recherche dont il va être question ci-dessous est centrée sur un dispositif de formation des maîtres à l'enseignement des mathématiques, dispositif pensé dans le cadre des théories didactiques. Certains aspects de cette étude ont été réinvestis par la suite bien sûr par son auteur à Montréal, mais également - entre autres - dans le cadre de la formation en didactique des mathématiques des enseignants primaire à la Faculté de Psychologie et des Sciences de l'Education de Genève. Nous nous centrerons ici uniquement sur les conclusions de la recherche initiale menée à Genève, conclusions exposées par son auteur (Portugais, 1995).

\section{La recherche de Portugais}

Portugais dans sa thèse s'est intéressé à la formation des futurs instituteurs et pour les besoins de sa recherche, a monté une ingénierie didactique avec une importante composante relative au travail des erreurs. Il pose le problème de l'enseignement de la didactique aux futurs instituteurs, en le formulant ainsi:«Comment faire pour former les futurs instituteurs à l'enseignement des mathématiques sans se limiter à leur offrir une simple fréquentation des contenus mathématiques et didactiques» (Portugais, 1995, p. 9)

En particulier il montre la nécessité d'apprendre à réellement travailler les erreurs des élèves et ce, du fait de la fonction même d'un enseignant qui est que l'élève apprenne. Nous avons vu ci-dessus que la réponse à l'erreur, en termes d'action professorale, pose un vrai problème. C'est dans l'optique d'une réflexion sur la formation que Portugais aborde la question du travail de l'erreur. Nous ne rentrerons pas ici dans les détails du dispositif complexe monté par l'auteur pour travailler cette question. Disons simplement qu'après une partie consacrée en séminaire à un travail d'analyse préalable du contenu spécifique à enseigner (selon la méthodologie classique de l'ingénierie didactique, à ce sujet voir Artigue 1988), les étudiants conçoivent, réalisent et analysent des séquences didactiques. Le dispositif permet un travail en duo et se reproduit à trois reprises dans un dispositif en boucles. Le séminaire est l'occasion d'aborder à propos du contenu choisi, procédures de calcul et erreurs des élèves. Portugais définit alors sa position relativement à la notion d'erreur d'élève: il reprend les distinctions faites par Salin (1976):

- d'une part la distinction entre échec s'opposant à succès, et erreur s'opposant à vérité,

- d'autre part celle faite entre point de vue de l'élève et point de vue du maître, ce dernier disposant, contrairement à l'élève, d'un système de validation de l'action lui permettant de lire les réussites et les erreurs de l'action de l'élève. Ce système de validation des actions engagées, s'il est absent, ne permet que l'approche binaire réussite/échec. 
C'est d'ailleurs pour cette raison que seuls les élèves «forts» peuvent produire une lecture didactique des erreurs soumises à leur sagacité comme le constate Büchli (2005) dans la recherche précédemment citée.

Qu'est censé faire l'étudiant en formation pour "travailler» les erreurs des élèves ? Deux étapes sont identifiées dans cette recherche qui concerne les algorithmes de calcul: l'une d'elle consiste à faire le diagnostic de l'erreur, l'autre consiste à travailler sur cette erreur.

En situation de classe, l'enseignant doit pouvoir d'abord repérer les erreurs des élèves: il s'agit donc pour lui d'une phase transitoire permettant l'intervention didactique ultérieure. Cette intervention didactique se fera en lien avec les analyses préalables.

Deux sortes de stratégies d'identification des erreurs sont mises en évidence chez les enseignants en formation. Les unes s'appuient sur la comparaison entre la production erronée de l'élève et un fonctionnement normal, sans erreur, qui sert de référent à l'identification de l'erreur. L'auteur analyse ce type de stratégie comme significatif de l'absence de prise en compte des analyses préalables. Les autres stratégies sont elles, centrées sur le fonctionnement erroné et sont donc faites en lien avec les analyses préalables en tant qu'elles fournissent des indications sur les erreurs potentielles.

L'intervention de l'enseignant -ici en formation- lorsqu'elle fait suite au repérage de l'erreur, n'est pas considérée comme une simple action en réponse à l'erreur de l'élève mais comme un véritable travail de l'erreur. "Nous emploierons l'expression "stratégie du travail de l'erreur plutôt qu'activité de traitement» pour désigner ce travail sur l'erreur afin de bien marquer le caractère didactique hautement finalisé des interventions du formé sur l'erreur» (Portugais, 1995, p. 166).

Il s'agit dit l'auteur d'un travail sur l'erreur qui se fait à partir de l'identification de l'erreur. C'est un processus qui vise à «exercer un contrôle sur l'activité de l'élève». Les stratégies mises en place par les enseignants en formation sujets de la recherche sont de deux types: celles qui opèrent sur le contrôle des actes $(F)$ et celles qui opèrent sur le contrôle du sens $(G)$.

L'opposition entre les formes $F$ et $G$ peut être ainsi formulée:

F repose sur la volonté (fiction ?) d'éradiquer l'erreur; F nécessite un changement de comportement et renforce l'apprentissage des règles, ce en quoi elle procède de l'associationnisme et de l'empirisme.

$G$ vise le contrôle conceptuel de l'activité mathématique; $G$ nécessite un changement au niveau conceptuel et s'oriente plutôt sur les significations sous-jacentes à la conduite des étapes de l'algorithme, ce en quoi elle procède d'une épistémologie essentiellement constructiviste (Portugais, 1995, p. 171).

L'auteur a pu observer que, suite à son dispositif, les stratégies utilisées par les formés pour travailler l'erreur sont de plus en plus complexes et de plus en plus pertinentes. Le dispositif proposé pour la formation articulant séminaire/ingénierie permet une évolution sensible et positive des choix didactiques opérés par les fu- 
turs enseignants en formation et une meilleure articulation entre théorie et pratique se fait jour chez les formés.

Si dans la recherche citée, la prise en compte de la dimension conceptuelle de l'activité mathématique s'accroit au cours de la formation, y compris dans le traitement des erreurs, il faut en chercher la raison dans la cohérence conceptuelle de l'ensemble du dispositif de formation. Les positions épistémologiques relatives à l'erreur, les anticipations et analyses des erreurs des élèves sur les contenus reposent sur les travaux de recherche de l'équipe de Genève. (Brun, Conne \& Retschitzki 1989, Brun \& Conne 1993, Brun, Conne, Lemoyne, \& Portugais, 1994). Dans le cadre de la théorie des champs conceptuels de Vergnaud, l'équipe de didactique de Genève sous la direction de Brun a étudié la question des algorithmes de calcul. L'analyse des erreurs est alors privilégiée comme moyen d'étude. La théorie des champs conceptuels est centrée sur la notion de schème, et a permis à l'équipe de revisiter la théorie de Van Lehn (Repair Theory). Affirmant qu'une erreur n'est pas nécessairement associée à une impasse, Brun a alors proposé que, dans le cadre de la théorie de Vergnaud, l'exécution d'un algorithme soit lue comme une recherche active d'adaptation à une situation de calcul au moyen des schèmes disponibles.

En particulier et c'est un point à retenir, quand il s'agit de la formation des enseignants, erreur n'est pas synonyme d'absence de connaissance.

La plupart des erreurs révèlent moins des absences de connaissance que des organisations originales de connaissances et de règles; celles-ci sont encore inadaptées à toutes les exigences du calcul à résoudre, mais elles peuvent être pertinentes par ailleurs, dans d'autres organisations et dans d'autres situations (Brun \& Conne, 1993, p. 30).

Ces travaux ont également donné lieu à l'établissement d'une typologie des erreurs de division que Portugais réutilise dans son dispositif de formation.

\section{Des propositions pour la formation}

De la recherche précédemment citée, nous retenons deux axes de réflexion relativement à la formation des enseignants. D'une part, et nous l'avons souligné, l'importance du travail d'analyse préalable à l'aune duquel seront identifiées et travaillées les erreurs produites. D'autre part, la nécessité d'une théorie qui permette de prendre en compte la dimension conceptuelle.

Les analyses anticipatrices des erreurs doivent prendre en compte les travaux permettant d'établir a priori les erreurs liées au savoir étudié. Nous ne reviendrons pas sur cet aspect développé dans le travail de Portugais (1995). Ce que nous voulons souligner ici est un autre axe de réflexion, celui de la temporalité didactique 7 . Chevallard (1985/1991) a mis en évidence que le rapport savoir durée est un élément fondamental du processus didactique. La temporalité didactique, dans son rapport avec le savoir, est à prendre en compte sous deux formes, l'une est liée à la chronogenèse du savoir, l'autre à sa topogenèse. 
La première qui a trait au déroulement temporel de l'enseignement/apprentissage nécessite un double point de vue. Le premier, «local», interroge la spécificité temporelle dans laquelle, du point de vue des connaissances, se situe la séquence d'enseignement en jeu. Le deuxième, plus macro, questionne l'inscription temporelle sur le long terme, l'enchaînement -notamment la connexité des séquences et des connaissances en jeu.

Autrement dit, ce n'est pas la seule tâche scolaire proposée qui est en question lorsqu'il s'agit d'anticiper, de pointer, d'analyser, etc.les erreurs mais c'est l'ensemble de la situation - y compris temporellement-, voire la macro situation d'apprentissage. Un concept devient alors pertinent pour enrichir la réflexion sur le travail de l'erreur c'est le concept de mémoire didactique.

L'enseignement ne peut pas fonctionner sans mémoire. Il compte, bien sûr, sur la mémoire des acteurs, c'est-à-dire sur les mémoires "personnelles» des élèves et des maîtres, celles dont ils disposent en tant que sujets humains. Elles sont sollicitées au cours des différentes phases de l'enseignement. Mais en outre, il y a une inscription de la mémoire dans le système didactique lui-même. Le système didactique ne peut pas laisser la gestion de la mémoire au seul niveau de ces mémoires personnelles. Il doit donc dans le traitement qu'il effectue du savoir, recueillir et prévoir des éléments de mémoire, au vu et au su de tous (élèves et maîtres) (Rouchier, 1991, pp. 29-30).

Ce concept a été défini dans le cadre de la théorie des situations par Brousseau et Centeno (1991). «Doter un organisme d'une mémoire lui permet de surseoir à certaines décisions sans perdre l'information susceptible de l'influencer et ainsi de ramener à l'intérieur de ses capacités de traitement des conditions qui auraient tendance à en sortir» (Brousseau \& Centeno, 1991, p. 199). Ces derniers ont alors travaillé la mémoire didactique de l'enseignant, la dimension propre au soussystème élève a été travaillée plus tardivement (Flückiger \& Mercier 2002, Flückiger 2005).

La mémoire didactique de l'enseignant va lui permettre de jouer un rôle d'activation des connaissances non encore transformées par l'élève en savoir décontextualisé, de jouer la carte de la chronogenèse. Ceci s'effectue par le biais de rappels des causes d'apprentissage, soit sous forme d'évocation des conditions et situations d'apparition initiale de ces connaissances, soit sous forme de reproduction de ces causes. La mémoire des faits et situations étant peu contrôlable, la mémoire didactique de l'enseignant va compenser cet obstacle en utilisant des informations contextualisées qui vont déterminer différents statuts aux connaissances en jeu. Pour l'élève également, la dimension mémorielle est à prendre en compte

La mémoire du système didactique [...] permet d'élargir les possibilités d'action de l'élève au-delà des savoirs et des connaissances qu'il peut mobiliser tout seul. La mémoire didactique agirait, en ce qui concerne l'enfant, dans cet espace que Vygotski appelle 'zone de développement proximale' lui donnant la possibilité d'utiliser des savoirs contextualisés, vécus avec le maître, et non encore codifiés dans sa mémoire personnelle (Brousseau \& Centeno, 1991, p. 201). 
Il est clair qu'un enseignement sans mémoire ne peut traiter les conceptions transitoires des élèves, les erreurs ne peuvent alors qu'être corrigées immédiatement et définitivement. La formation des enseignants ne peut ignorer ce concept et ses implications.

Concernant la temporalité didactique, le deuxième axe à considérer est lié au processus de conceptualisation. Le temps de l'enseignement n'est pas superposable au temps de l'apprentissage, de plus, le temps de l'apprentissage n'est ni linéaire ni cumulatif or les décisions de remédiation donnent à voir cette conception implicite erronée. Là aussi la formation doit mettre en place des dispositifs qui invalident cette position. En particulier, cela sous-entend la prise en compte des états différents d'élaboration de réseaux conceptuels dans une classe à un moment donné et ce, même si l'enseignement prodigué a été le même. Il y a, du point de vue de la gestion professorale, une difficulté relativement à ces différences: le cas de l'erreur n'étant qu'un des cas possibles d'existence de positions contradictoires. Rappelons que dans les propositions d'action des enseignants en formation, l'une d'elle consiste à provoquer un débat pour traiter une erreur: ce n'est pas étonnant, débattre, mettre en commun, sont des injonctions fortes dans les prescriptions pédagogiques actuelles. Qu'il s'agisse de confrontation avec des erreurs ou des contradictions internes à la classe ou à un individu, la gestion de ces contradictions n'est pas simple. Cela nécessite une formation et donc une théorie permettant de penser ces contradictions. Ratsimba-Rajohn (1992) définit ce qu'il appelle "une macle de contradictions». C'est, dit-il, le système dans lequel se meut un élève, lorsque celui-ci est formé de recettes ou de procédés juxtaposés dont certains sont contradictoires entre eux; or ces contradictions ne sont pas nécessairement perçues par l'élève. De ce fait il ne perçoit pas non plus les erreurs, ni les paradoxes qui en découlent. Reprenant l'idée générale que l'élaboration des connaissances passe par des rectifications, des rejets d'anciennes conceptions, des conflits, etc. l'auteur affirme que l'idée de macle de contradiction est sous-jacente à toute production de connaissance. Se pose alors la question de la gestion de ces contradictions; or, une gestion de séances d'enseignement qui ne considère pas les macles de contradiction entraîne une utilisation de l'ostension ${ }^{8}$ dit Ratsimba-Rajohn (1992). Et dans ce cas, la connaissance est considérée par l'enseignant comme un système d'affirmations à transférer plutôt que comme une macle de contradictions à dénouer éventuellement avec son aide: on est alors loin de la perspective constructiviste de l'apprentissage.

\section{En conclusion}

Dans une perspective didactique la question du savoir en jeu est centrale. Relativement aux erreurs, il faut donc, en amont des séquences d'enseignement, travailler le savoir pour en faire une analyse a priori qui tienne compte des erreurs 
potentielles liées à la fois au savoir et à la situation qui va lui donner sens. Le repérage et le traitement des erreurs vont dépendre de la qualité des anticipations ainsi faites. Prendre en compte la dimension non linéaire, les nécessaires réorganisations dans la structuration des connaissances non pas comme un écart à la norme mais comme inhérente à la conceptualisation en cours est un autre aspect à mettre en évidence dans la formation à l'enseignement.

Autre point que nous voulons souligner pour terminer, l'absolue nécessité mais la grande difficulté de la gestion des contradictions quand il s'agit d'apprentissage et tout particulièrement du travail de l'erreur: contradictions internes à l'individu qui apprend, contradictions entre les élèves d'une classe. L'une des entrées possibles pour penser cette question difficile quand il s'agit de formation est celle de la mémoire didactique, celle du sous système enseignant aussi bien que celle du sous système élève.

Penser les apprentissages à la fois dans la durée et dans la non linéarité est certainement une des clés de lecture de la réalité didactique qui peut permettre, dans la formation, l'évolution des pratiques spontanées des enseignants relativement à la question de l'erreur.

\section{Notes}

1 Expression reprise notamment de Portugais (1995)

2 Dans le texte, pour alléger l'écriture et sauf mention contraire, le terme «didactique» renverra à ce courant de didactique des mathématiques francophones

3 Le terme de "conduite " indique la prise en compte à la fois du comportement et des représentations.

4 Nous sommes davantage proches de l'idée de stratégie inconsciente développée par Bourdieu, que d'une stratégie pensée comme un choix conscient.

5 Brousseau dans son glossaire de quelques concepts de la théorie des situations (web 2003) définit les situations a-didactiques comme un certain type de situation didactique qui ménage des situations partiellement libérées d'intervention directes.

6 Utilisés par Chevallard dans «La transposition Didactique» (1985), les termes de chronogenèse et de topogenèse sont là pour différencier deux axes d'évolution du savoir lors de l'apprentissage. Le premier fait référence à la diachronie du système didactique, le deuxième au système de places différenciées qu'occupent l'enseignant et l'enseigné relativement au savoir. En utilisant ces termes, Brousseau \& Centeno (1991) mettent l'accent sur deux structurations du savoir, l'une sémantique liée à la chronogenèse du savoir l'autre syntaxique en lien avec la topogenèse.

7 Buchli (2005) montre à propos de l'analyse des erreurs que font les élèves eux-mêmes, que seuls ceux de statut scolaire fort ou moyen prennent en compte la dimension temporelle de l'enseignement/apprentissage. 


\section{Références bibliographiques}

Amigues, R. (1991). L'apprenti, l'erreur, le système. Interactions didactiques, 12, 9-25.

Artigue, M. (1988). Ingénierie didactique. Recherches en Didactique des Mathématiques, 9 (3), 281-308.

Astolfi, J. P. (1997). L'erreur un outil pour enseigner. Paris: ESF Editeur.

Brousseau, G. (1980). Problème de l'enseignement des décimaux. Recherches en didactique des mathématiques, 1 (1), 11-58.

Brousseau, G. (1986). Fondements et méthodes de la didactique des mathématiques. Recherches en didactique des mathématiques, 7 (2), 33-115.

Brousseau, G. (1990). Le contrat didactique : le milieu. Recherches en didactique des mathématiques, 9(3), 309-336.

Brousseau, G. (1998). Théorie des situations didactiques. Grenoble: La Pensée Sauvage Editions.

Brousseau, G. (2000-2001). Les erreurs des élèves en mathématiques. Etude dans le cadre de la théorie des situations didactiques. Petit x, 57, 5-30.

Brousseau, G. (2003). L'enseignement des mathématiques dans la scolarité obligatoire: micro et macro didactique. [Article Web non publié en français ].

Brousseau, G., \& Centeno, J. (1991). Rôle de la mémoire didactique de l'enseignant. Recherches en didactique des mathématiques, 11 (2/3), 167-210.

Brun, J. (1999). A propos du statut de l'erreur dans l'enseignement des mathématiques, Résonances, 5, 7-9.

Brun, J., Conne, F. \& Retschitzki, J. (1989). L'étude des algorithmes de calcul dans la transmission et la constitution des connaissances numériques. Projet de recherche FNRS (Rapport final 1991). Genève, FPSE.

Brun, J. \& Conne, F. (1993). Calculs et erreurs systématiques. Journal de l'enseignement primaire, Genève, 43, 29-31.

Brun, J., Conne, F., Lemoyne, G., \& Portugais, J. (1994). La notion de schème dans l'interprétation des erreurs des élèves à des algorithmes de calcul écrit. Cahiers de la recherche en éducation, 1 (1), 117-132.

Büchli, T. (2005). Approche didactique des représentations de l'erreur en mathématiques chez les élèves de 6P. Mémoire de licence en Sciences de l'Education, Université de Genève.

Chevallard, Y. (1985/1991). La transposition didactique. Du savoir savant au savoir enseigné. Grenoble: La Pensée Sauvage Editions.

Chevallard, Y. (1999). L'analyse des pratiques enseignantes en théorie anthropologique du didactique. Recherches en didactique des mathématiques, 19 (2), 221-266.

Comiti, C. \& Grenier, D. (1991). Objectifs et méthodes pour une recherche sur les représentations d'enseignants de mathématiques. Texte présenté à la VIème école d'été de Didactique des Mathématiques, Plestin les Grèves.

Flückiger, A. (2005). Macro-Situation and Numerical Knowledge Building: The Role of Pupils' Didactic Memory in Classroom Interactions. Educational Studies in Mathematics, 59 $(1 / 2 / 3), 59-84$.

Flückiger, A. \& Mercier, A. (2002). Le rôle d'une mémoire didactique des élèves, sa gestion par le professeur. Revue Française de Pédagogie, 141, 27-35.

Leplat, J. \& Pailhous, J. (1974). Quelques remarques sur l'origine des erreurs. Bulletin de psychologie, 27, 729-736.

Margolinas, C. (1993). De l'importance du vrai et du faux dans la classe de mathématiques (p. 170). Grenoble: La Pensée Sauvage Editions.

Portugais, J. (1995). Didactique des mathématiques et formation des enseignants. Berne: Peter Lang.

Ratsimba-Rajohn, H. (1992). Contribution à l'étude de la hiérarchie implicative. Application à l'analyse de la gestion didactique des phénomènes d'ostension et de contradiction. Thèse d'université en mathématiques et applications, Université de Rennes I. 
Ravenstein, J. \& Sensevy, G. (1993-1994). Statuts de l'erreur dans la relation didactique. Grand N, 54, 83-90.

Rouchier, A. (1980). Présentation. Recherches en didactique des mathématiques, 1 (1), 7-11.

Rouchier, A. (1991). Etude de la conceptualisation dans le système didactique en mathématiques et informatique élémentaires: proportionnalité, structures itérativo- récursives, institutionnalisation (pp. 29-30). Thèse de doctorat d'Etat en didactique des mathématiques, Université d'Orléans.

Salin, M. H. (1976). Le rôle de l'erreur dans l'apprentissage des mathématiques à l'école primaire. Mémoire de DEA en Didactique des mathématiques, IREM de Bordeaux.

Vergnaud, G. (1991). La théorie des champs conceptuels. Recherche en didactique des mathématiques, $10(2 / 3), 133-170$.

Mots clés: didactique des mathématiques, erreur, traitement de l'erreur, formation des enseignants, mémoire didactique

\section{Lernen durch die Arbeit am Fehler und die Mathematikdidaktik}

\section{Zusammenfassung}

Die Entwicklung der konstruktivistischen Theorien des Lernens hat die Auffassungen über die Bedeutung des Fehlers für die Entwicklung des Wissens verändert. Die didaktischen Theorien im Allgemeinen und die Mathematikdidaktik im Speziellen haben sich daran beteiligt. Dieser Artikel zeigt auf, auf welche Art und Weise die Mathematikdidaktik zu dieser Entwicklung beigetragen hat, und wie einige der im Laufe der letzten dreissig Jahre entwickelten Konzepte die Diskussion um die Problematik des Fehlers im Unterricht anhand des schulischen Mathematiklernens vorantreiben. Daten, die an Lehramtsstudierenden erhoben wurden, verweisen auf Differenz zwischen dem bestehenden konstruktivistischen Diskurs und den reellen Praxen im Umgang mit Fehlern. Der Artikel stellt sich abschliessend dem Problem, wie eine Lehrer/innenbildung auszusehen hätte, die sich der Weiterentwicklung dieser Praktiken widmet, und schlägt dazu einige Leitlinien vor.

Schlagworte: Mathematikdidaktik, Fehler, Fehlerkultur, LehrerInnenbildung, didaktisches Gedächtnis, Konstruktivismus

\section{Formazione al lavoro con l'errore e didattica della matematica}

\section{Riassunto}

Lo sviluppo delle teorie costruttiviste ha modificato il rapporto con l'errore nel contesto dello sviluppo delle conoscenze. Le teorie didattiche in generale e quelle della didattica della matematica in particolare sono state parte di questa evolu- 
zione. L'articolo mostra quale sia stato il contributo di quest'ultima e come certi concetti da essa sviluppati nel corso degli ultimi trent'anni permettano di arricchire l'insegnamento/apprendimento della matematica a scuola. Muovendo dai dati raccolti presso gli insegnanti in formazione, si evidenzia il divario che esiste tra il discorso che abitualmente considera la prospettiva costruttivista e le pratiche reali di gestione dell'errore. L'articolo conclude ponendo il problema di una formazione degli insegnanti in grado di far evolvere questa problematica e formula alcune proposte in merito.

Parole chiave: didattica della matematica, errore, trattamento dell'errore, formazione degli insegnanti, memoria didattica

\section{Training on error handling, and the didactics of mathematics}

\section{Summary}

The development of constructivist theories has changed the relation to the concept of error when considered in connection with the development of knowledge. The didactical theories in general and particularly contributed to this trend. This article explains how the latter contributed to this evolution and how some of the concepts it developed over the past thirty years have enriched the error issue when considered in the context of mathematics learning at school. Based on data collected from students in educational sciences, the article highlights the discrepancy that exists between, on the one hand, the usual discourse taking into account the constructivist perspective and, on the other hand, the actual practices as implemented when dealing with errors. It raises the issue of designing teacher trainings that would permit to evolve these practices, and proposes several avenues for thinking this training up.

Key words: mathematical didactic, error, teacher's action, training of teachers, didactic memory 
$\mathrm{T} \mathrm{h}$ e $\mathrm{m} \mathrm{a}$ 